\title{
Web Query Behaviour Concerning HEV (Blue) Light in Ophthalmology
}

DOI: https://doi.org/10.32007/jfacmedbagdad.6331840.

\author{
Ahmed ML. Al-Imam* \\ Hend J. Jasim ** \\ MSc (Dermatology), MRes (Neuroscience), MSc (Human Anatomy) \\ Loay M. Hassan *** \\ FICMS (Pediatrics), CABMS (Pediatrics) \\ CABMS (Ophthalmology), MRCS (Surgery)
}

This work is licensed under a Creative Commons Attribution-NonCommercial 4.0 International License.

J Fac Med Baghdad 2021; Vol.63, No.3

Received: April, 2021

Accepted: Aug., 2021

Published: Oct., 2021

Background: High-energy visible (HEV) possesses high-frequency in the violet-blue band of the visible light spectrum. Blue light has relevance to ophthalmology via photochemically-induced retinal injury.

Objectives: To explore the spatial-temporal mapping of online search behavior concerning HEV light.

Materials and Methods: We retrieved raw data of web search volume, via Microsoft Google Trends, using five search topics; "Biological effects of HEV light", "Vision impairment", "Macular degeneration", "Retinal tear", and "Retinal detachment", for the period 2004-2020.

Results: Web users, mainly from Far-East Asia and Australasia, were most interested in seeking online information concerning "Macular degeneration" and "Vision impairment" search topics, moderately interested in "Biological effects of HEV light" and "Retinal detachment", and least interested in "Retinal tear". Internet users from the Middle East and Arab world contributed minimally to the holistic map. Web queries increased during 2004-2006 and 2018-2020; however, these trends were respondents to specific search topics. For instance, the search volume for the "Biological effects of HEV light" increased significantly in 2018-2020. Predictive modeling was most accurate for "Biological effects of HEV light". The strongest correlation was for "Vision impairment" versus "Macular degeneration". ANOVA, linear modeling, and machine learning unanimously agreed on predictors' significant effect (search topics and time) on the web search volume.

Conclusion: Web queries' mapping provided indirect evidence on the potential causality between HEV light and retinal damage. Future research mandates rigor observational and experimental studies.

Keywords: Blue light; high-energy visible light; macular degeneration; photochemically-induced retinal injury; retinal detachment; retinal perforation; retinal tear.

\section{Introduction:}

High-energy visible light (HEV), also known as blue light, is a high-energy and high-frequency light in the violet-blue band, 400-450 nm wavelength, of the visible light spectrum; it potentially has several biological effects, including those on the eye and the retina [1]. A body of medical evidence incriminates HEV light as an agent of retinal injury and macular degeneration $[1,2]$. In 2014, a trio of scientists from Japan and the United States received the Nobel Prize in Physics for inventing the blue light-emitting diodes (LEDs); professors Isamu Akasaki, Hiroshi Amano, and Shuji Nakamura developed the first blue LEDs in the early 1990s, which enabled a novel generation of bright, energy-efficient white lamps, and color LED displays [3]. Blue LED light sources are becoming abundant in today's life; exposure to blue light originates from various devices, including computers, televisions, and cell phones [1-3].

* Department of Anatomy and Cellular Biology, College of Medicine, University of Baghdad, Email: ahmed.mohammed@comed.uobaghdad.edu.iq.

** Department of Pediatrics, Al-Batool Teaching

Hospital, Email: hendaldoori0571@gmail.com.

*** Department of Ophthalmology, Baquba Teaching

Hospital, Email: drloaymahmod999@gmail.com.
Blue light hazards manifest from its potential to induce an irreversible photochemically-induced injury to the retina due to the exposure to electromagnetic radiation at wavelengths primarily between 400 and $450 \mathrm{~nm}$ [1, 2]. Further, a photochemically-induced retinal injury arises from oxidative stress, inflammatory apoptosis, mitochondrial apoptosis, DNA damage, and the absorption of blue light photons by the photoreceptors in the retina, resulting in dry eye disease, keratitis, glaucoma, and visual impairment; nonetheless, researchers have not thoroughly studied the phenomenon in humans, but inconclusively in some rodents' species, primates, and in vitro studies [1-4]. Some commercially available sunglasses and beauty creams can specifically block blue light $[1,4]$. Blue cut lenses, spectacles, and screen filters feature a special coating that reflects harmful blue light and prevents it from passing through the eye to the retina [4]. Blue light filtering apparatuses can partially filter blue light without significantly degrading the visual performance; these lenses can serve as a supplemental measure for protecting the retina from $\mathrm{HEV}$ light hazards [4, 5]. However, the ultraviolet and high-energy violet-blue (400 to $450 \mathrm{~nm}$ ) light-filtering capacity varies across different products and manufacturers [5]. As a primary 
objective, we aim to explore the spatial (geographic) mapping and the temporal (chronological) mapping of the period 2004-2020, of web search volume for specific search topics related to the biological effects of HEV light and the photochemically-induced retinal injury. As a secondary objective, we aim to evaluate the causality relationship, given the Bradford Hill criteria, between potential predictors (search topics and time) and the outcome (web search volume).

\section{Materials and Methods}

We retrieved raw data via the Microsoft Google Trends engine and conducted data analyses using IBM-SPSS version 24 and Microsoft Office Excel 2016 with Analysis ToolPak add-in. We deployed five search topics; "Biological effects of HEV light", "Vision impairment", "Macular degeneration", "Retinal tear", and "Retinal detachment" [6]. The study was conducted following the standard protocol of the Ethics and Scientific Committee of the College of Medicine at the University of Baghdad, the declaration of Helsinki by the World Medical Association, and the ethical principles of the Framingham consensus of 1997. We evaluated the level of evidence of the current study as per the categorization system rectified by the Oxford Centre for Evidence-Based Medicine [7].

\section{Results}

Online Information Seeking Behaviour: Concerning the spatial mapping, forty countries contributed to the holistic map, and the top twenty countries included Japan, New Zealand, Singapore, United Arab Emirates, Ireland, Thailand, Australia, Philippines, United States,
Indonesia, Malaysia, Vietnam, Canada, Greece, United Kingdom, South Africa, India, Pakistan, Denmark, and Norway. Nations from Far-East Asia and Australasia had the largest share among the former countries. On the contrary, only five countries from the Middle East and the Arab world contributed to the holistic map (12.5\%), including United Arab Emirates, Palestine, Egypt, Saudi Arabia, and Turkey. Concerning the temporal mapping, the web search volume, reflecting the interest of internet users in a specific web query, variated according to the search topics; "Biological effects of HEV light" (mean=14.29, standard error of mean=1.216, skewness $=2,700, \quad$ kurtosis $=7.628$, "Vision impairment" (38.63, 0.559, 1.192, 1.788), "Macular degeneration" (40.41, 0.706, 1.877, 3.929), "Retinal tear" $(1.79,0.056,2.964,19.262)$, and "Retinal detachment" (26.81, 0.241, 0.866, 1.157). Statistical outliers were evident during 2004-2006 for all the search topics except for "Biological effects of HEV light". Nonetheless, the latter had statistical outliers in 2018-2020 (Figure 1). Further, as per predictive modeling, the "Biological effects of HEV light" search query had the strongest measure of association among all search topics $\left(\mathrm{R}^{2}\right.$ score $=0.9471$, coefficient of correlation=0.9732). Accordingly, surface web users were most interested in "Macular degeneration" and "Vision impairment" search topics, moderately interested in "Retinal detachment" and "Biological effects of HEV light", and they were least interested "Retinal tear" (Figure 2). Online information search queries originated mainly from Far-East Asia and Australasia, while Middle-Eastern and Arab countries contributed minimally.

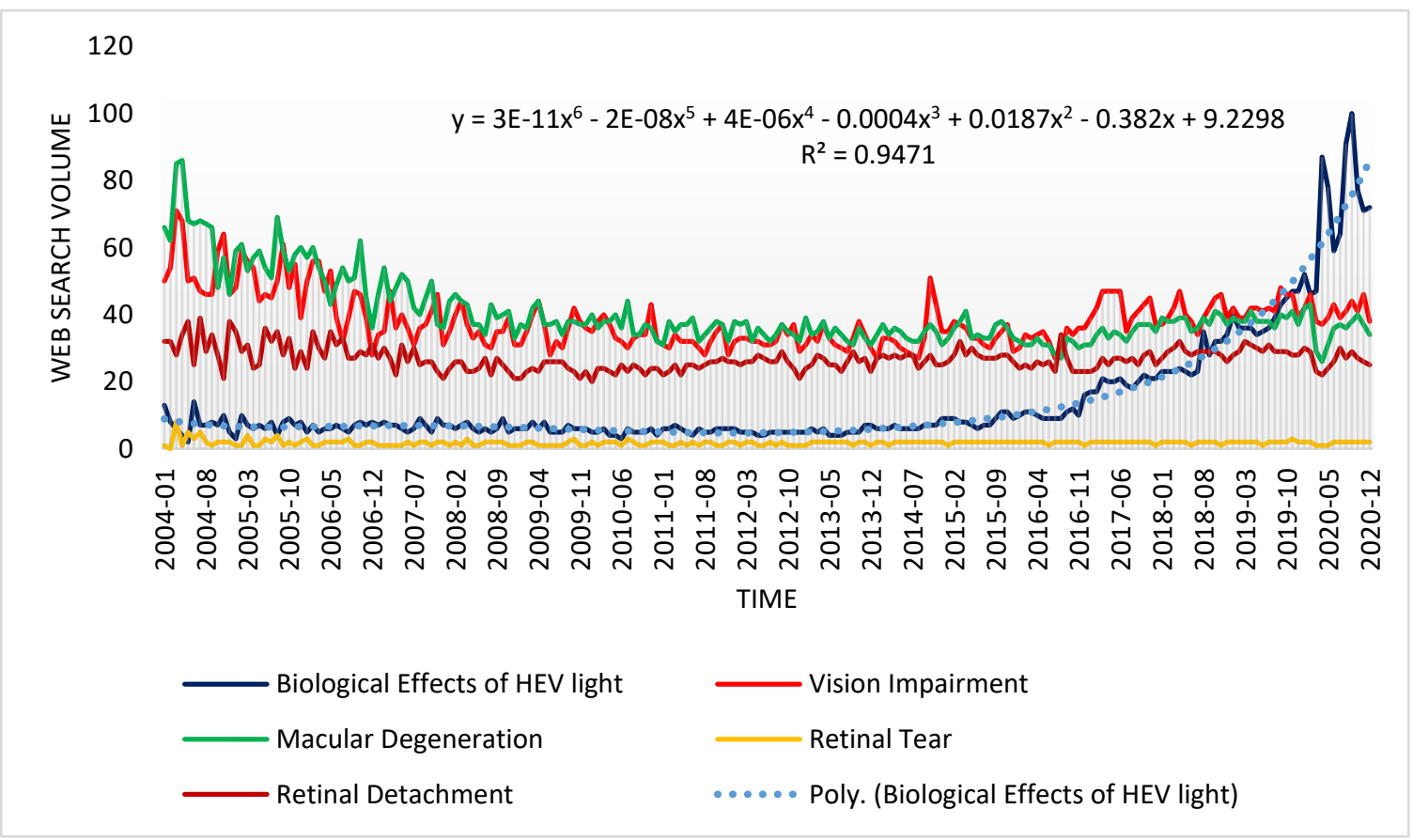

Figure 1. Temporal Mapping of the Web Search Volume. 
Related Online Information Seeking Behaviour: Related queries were not limited to "blue light glasses", "blue glasses", "blue light filter", "blue light blocking glasses", "blue light lenses", "computer glasses", "blue light LED", "retinal detachment surgery", "floaters", "glaucoma", "cataract", "eye floaters", "retinal tear surgery", "retinal tear laser surgery", "retinal hole", "macula", "macular degeneration test", "macular degeneration surgery", "lutein", "macular degeneration injections", "diabetes", "diabetic retinopathy", "causes of macular degeneration", "vitamins for macular degeneration", "visual diability", and "temporary vision loss". Most related web queries were relevant to ophthalmology, endocrinology, and biotechnology.

Predictive Models of Search Topics: We were successful in creating predictive models using the $6^{\text {th }}$ order polynomial function that describes the temporal patterns that govern each of the five search topics for the timespan 2004-2020. Each search topic had a large effect size except for "Retinal tear" search topic; "Biological effects of HEV light" (predictive equation: $\mathrm{y}=3 \mathrm{E}-11 \mathrm{x} 6-2 \mathrm{E}-08 \mathrm{x} 5+4 \mathrm{E}-06 \mathrm{x} 4-0.0004 \mathrm{x} 3+$ $0.0187 \times 2-0.382 \mathrm{x}+9.2298, \quad \mathrm{R}^{2}$ score $=0.9471$, coeffecient of correlation $=0.9732$, large effect size), "vision impairment" ( $\mathrm{y}=-3 \mathrm{E}-11 \mathrm{x} 6+2 \mathrm{E}-08 \mathrm{x} 5-4 \mathrm{E}-$ $06 \mathrm{x} 4+0.0004 \times 3-0.0126 \times 2-0.3185 \mathrm{x}+57.723$, $0.6206, \quad 0.7878$, large effect size), "macular degeneration" ( $\mathrm{y}=-3 \mathrm{E}-11 \mathrm{x} 6+1 \mathrm{E}-08 \times 5-3 \mathrm{E}-06 \times 4+$ $0.0002 \times 3-0.0032 \times 2-0.7644 x+71.536,0.8250$, 0.9083 , large effect size), "retinal tear" ( $\mathrm{y}=2 \mathrm{E}-12 \mathrm{x} 6$ $1 \mathrm{E}-09 \mathrm{x} 5+3 \mathrm{E}-07 \mathrm{x} 4-4 \mathrm{E}-05 \mathrm{x} 3+0.0025 \mathrm{x} 2-0.0972 \mathrm{x}+$ $3.1138,0.1097,0.3312$, medium effect size), and "retinal detachment" $(\mathrm{y}=-3 \mathrm{E}-11 \mathrm{x} 6+2 \mathrm{E}-08 \mathrm{x} 5-5 \mathrm{E}-$ $06 \times 4+0.0006 \times 3-0.0299 \times 2+0.4588 x+29.821$, $0.4051,0.6365$, large effect size).

Non-Bayesian Statistics: We ran a normality test, the Shapiro-Wilk test, for the total web search volume, for each of the search topics, and for each year from 2004 to 2020 . The search volume did not follow a normal distribution in all scenarios $(\mathrm{p}$-value $<=0.001)$. Therefore, we ran non-parametric testing, including Kendall's tau-b $(\tau b)$ correlation. We also ran a multifactorial (two-way) univariate analysis of variances (ANOVA), given its statistical robustness. Bivariate correlations among the five search terms detected some significant correlations, including "Biological effects of HEV light" versus "Vision impairment" (Kendall's tau-b ( $\tau b)$ correlation coefficient $=0.273, \quad p-$ value $<0.001$ ), "Biological effects of HEV light" versus "Retinal tear" (0.142, p-value $=0.015)$, "Biological effects of HEV light" versus "Retinal detachment" (0.182, p-value $<0.001)$, "Vision impairment" versus "Macular degeneration" (0.466, p-value<0.001), "Vision impairment" versus "Retinal detachment" (0.284, p-value $<0.001)$, "Macular degeneration" versus "Retinal detachment" $(0.301$, p-value $<0.001)$, and
"Retinal tear" versus "Retinal detachment" $(0.144$, pvalue $=0.013)$. Bivariate correlations were in the positive direction, and all had a small effect size except for "Macular degeneration" versus "Vision impairment" and "Macular degeneration" versus "Retinal detachment"; each had a moderate effect size. Concerning the univariate analysis of variance, we fed the two-factor ANOVA model with the search topics and time as model predictors (independent variables), and the web search volume as the outcome (dependent variables). The model was statistically significant with a large effect size $(\mathrm{df}=84$, mean sqaure $=3607.003$, $\mathrm{F}=236.012$, $\mathrm{p}$-value $<0.001$, partial eta $^{2}=0.955$, adjusted $\mathrm{R}^{2}=0.951$ ). The model's predictors were statistically significant; search topic $(\mathrm{df}=4$, mean sqaure $=$ 54983.841, $\mathrm{F}=3597.676, \quad \mathrm{p}$-value $<0.001$, partial eta $\left.{ }^{2}=0.939\right)$ and time $(\mathrm{df}=15$, mean sqaure $=1455.297$, $\mathrm{F}=$ 95.222, $\mathrm{p}$-value $<0.001$, partial $\mathrm{eta}^{2}=0.620$ ). Accordingly, each of the five search topics had a significant effect on the web search volume. The posthoc analysis using Tukey's HSD validated statistically significant differences for multiple comparisons, i.e., between the search topics (p-value<0.001) (Figure 2).

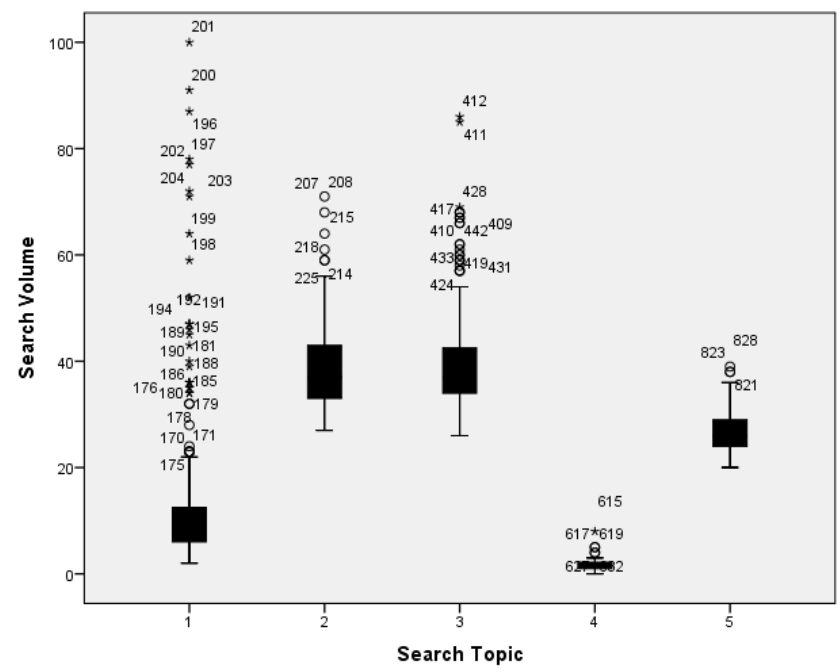

Figure 2. Boxplot Presentation of the Web Search Volume.

* X-axis numerals (1-5) represent the search topics (web queries); (1) "Biological effects of HEV light", (2) "Vision impairment", (3) "Macular degeneration", (4) "Retinal tear", and (5) "Retinal detachment".

Further, we conducted automatic linear modeling with a similar design of the univariate analysis of variance concerning the model's predictors and outcome. The model deployed automatic data preparation, a forward stepwise selection method, at an information criterion 4,401.399 (Figure 3). Similar to the ANOVA test, automatic linear modeling detected a significant effect for the search topics (predictor's importance $=0.908, \mathrm{p}$ value $<0.001)$ and time $(0.092,0.001)$ (Figure 3 and 4$)$. 


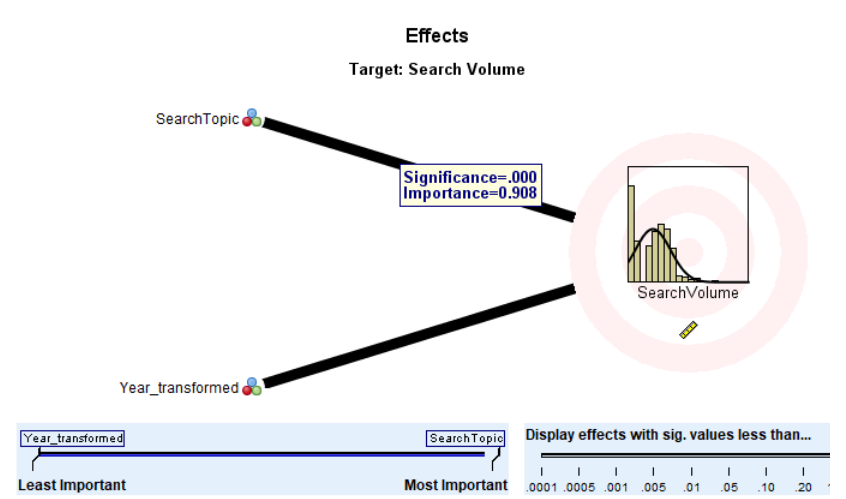

Figure 3. Automatic Linear Modeling: Summary of Significant Predictors.

Supervised Machine Learning: We implemented multilayer perceptron neural networks and scaled conjugate gradient optimization algorithm, and a default SPSS allocation of the training set and testing set at $70 \%$ and $30 \%$ of the whole dataset, respectively. The neural networks yielded synaptic weights and independent variables importance analysis. The neural network also possessed an analogous architecture of the ANOVA test and the automatic linear model, using the same predictors and outcome. The neural network assigned higher importance for search topics (predictor's importance $=0.600, \quad$ normalized importance $=100 \%)$ than time $(0.400,66.8 \%)$.

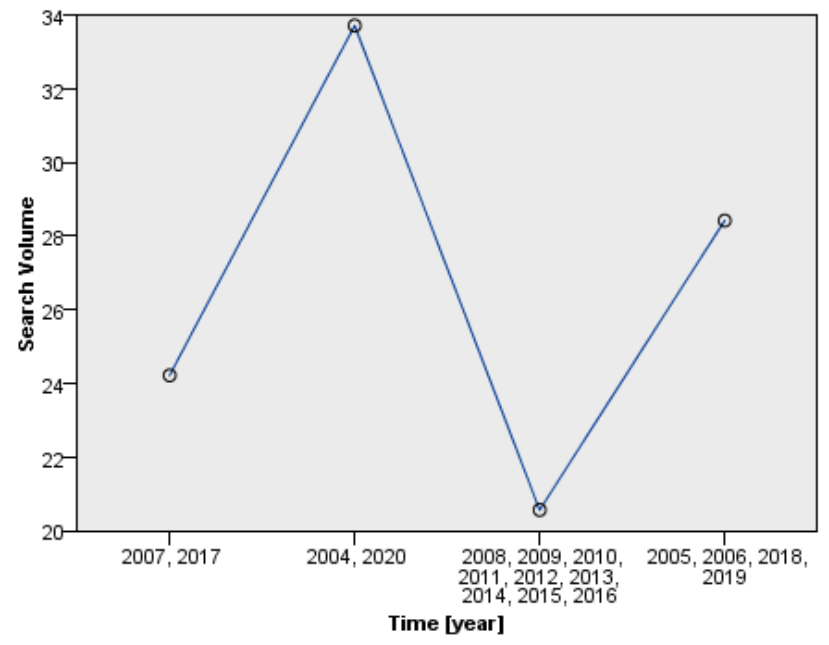

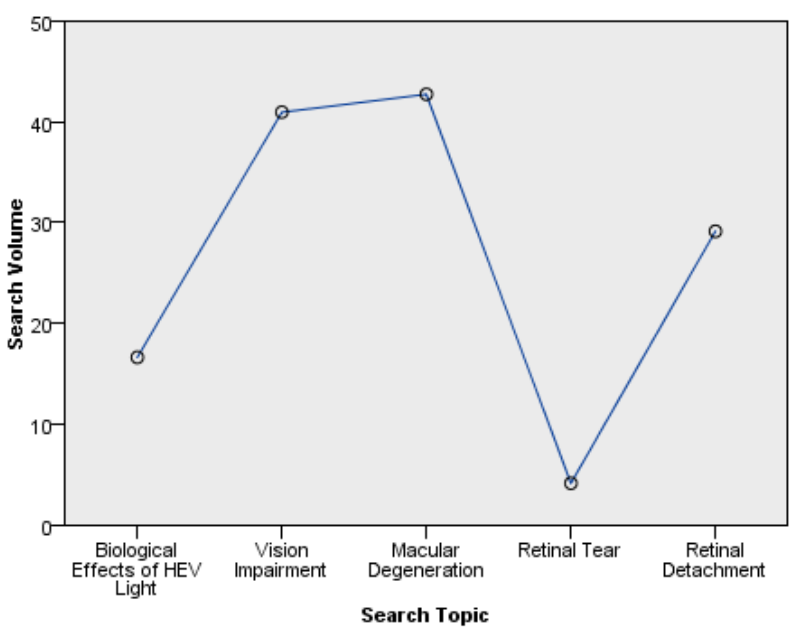

Figure 4. Automatic Linear Modeling: Estimated Means of Search Volume

* Estimated means of search volume by "time [years]" and "search topics".

\section{Discussion}

Users of the internet (surface web) were most interested in seeking online information concerning "Macular degeneration" and "Vision impairment" search topics, moderately interested in "Biological effects of HEV light" and "Retinal detachment", while being least interested in "Retinal tear". Most internet users were from Far-East Asia and Australasia, while those from the Arab world and the Middle East contributed minimally to the holistic spatial map. Web queries increased during 2004-2006 and 2018-2020; however, these trends were respondents to specific search topics. For instance, the web search volume for the "Biological effects of HEV light" increased beyond 2014 and significantly in 2018-2020, perhaps following the announcement of the Nobel Prize in Physics for inventing blue LEDs. The other four search topics' queries were most prominent in 2004, 2005, and 2006 (Figure 1). The significant rise in the web search volume of "Biological effects of HEV light" in 20192020 may also relate to the severe acute respiratory syndrome coronavirus 2 (SARS-CoV-2), also known as the Coronavirus disease 2019 (COVID-19) [8-10]. During the pandemic, people may adapt to the social anomie using different methods; conformity, innovation, ritualism, retreatism, and rebellion [11]. For instance, virtual social media, including Facebook, Whatsapp, and Instagram, represent an alternative to the social interaction in everyday life that became restricted or entirely banned by frequent lockdowns due to the pandemic. Accordingly, the unprecedented approach to life enforces a substantial increment in the number of hours an individual may spend using his mobile phone or in front of a computer screen, leading to eye strain and more exposure to the harmful HEV light. Most related web queries were relevant to ophthalmology, optometry, endocrinology, traumatology, and 
biotechnology. Predictive modeling was most accurate for the search topic "Biological effects of HEV light" and least accurate for the search topic "Retinal tear". The strongest bivariate correlation was for "Vision impairment" versus "Macular degeneration", and the weakest was for "Biological effects of HEV light" versus "Retinal tear". The univariate analysis of variance, automatic linear modeling, and supervised machine learning unanimously agreed on predictors' significant effect (search topics and time) on the web search volume, thus, reconciling frequentists (nonBayesian) statistics and machine learning. We attempted to evaluate the article's level of evidence as per the categorization system rectified by the Centre for Evidence-Based Medicine at the University of Oxford [7]. However, it was impossible to infer the former as our study represents an amalgam of data analytics, encompassing non-Bayesian statistical analysis, machine learning, a review of the literature with critical appraisal, and a case report. Nonetheless, future research on HEV light should incorporate studies of the supreme level of evidence (level-1 and 2), including randomized controlled trials, systematic reviews, and meta-analytic studies [12-15]. We recommend clinical research scientists to incorporate online information research behavior and digital epidemiology data to complement classical epidemiological and experimental knowledge concerning the high-energy visible light and photochemically-induced retinal injury. We encourage continuous medical development professionals to boost the awareness of healthcare practitioners, particularly ophthalmologists and optometrists, concerning the harmful medical consequences of blue lights, prophylactic measures, and across all age groups, including children. We prompt research methodologists and data scientists to attempt conforming frequentists statistics with machine learning as a modality of artificial intelligence to study this phenomenon [16, 17]. Finally, it is established that blue light, with an energy of 400-450 nm (wavelength), results in a harmful effect on vision; however, the process is time and dose-dependent and may take years to decades [1]. Besides, there is also a lack of high-level evidence concerning the duration of time that blue light needs to result in photochemically-induced retinal damage [1,2]. Nonetheless, it is recommended to use blue-light filtering spectacle lenses to block at least $90 \%$ of the high-energy visible blue light $[4,5]$. Accordingly, the authors find it mandatory to conduct subsequent randomized controlled trials, systematic reviews, and meta-analytic studies concerning our study objectives [7].

\section{Case Report of Interest}

An otherwise healthy, 37 years old male patient complained of eye floaters affecting and disturbing the visual fields of both eyes. The patient had an old history of blunt trauma affecting the left eye at the age of 18 years old, after which he noticed the appearance of floaters affecting the left eye's visual field. The condition did not progress any further, but in 2014, he decided to visit an ophthalmologist to evaluate his vision, and all tests and examinations were normal, including the integrity of the retina. Since 2016, the patient assumed a research position at an academic institute and worked long hours in front of a computer screen. In 2021, specifically in February, he noticed that the floaters affecting his left eye have increased, in addition to the appearance of new floaters affecting his right eye's visual field. Therefore, he visited a specialized ophthalmology clinic, and he underwent an examination at the optometrist; he had a normal intraocular pressure and a normal visual acuity (6/6). A specialist ophthalmologist also examined both eyes, and he found several retinal holes and tears affecting the superior temporal field of the left eye's retina, while the right eye's retina was normal. Accordingly, the ophthalmologist advised the patient to manage the condition using Laser (Argon Laser, $514 \mathrm{~nm}$ ) in three sessions, 2-3 weeks apart between consecutive sessions, as a prophylactic measure to prevent the retinal pathology from progressing to a retinal detachment.

The physician also advised the patient to reduce working hours on his computers and use spectacles to filter the HEV (blue) light. Following the third laser session, the patient developed blurred vision due to anticipated laser-induced retinal edema, which resolved spontaneously after three weeks, after which he revisited the optometrist and the ophthalmologist. The patient had normal intraocular pressure, normal visual acuity (6/6), and a stable retina. The ophthalmologist asserted that no further intervention is required, but only follow-up visits. The case provides anecdotal evidence on the harmful effects of the HEV (blue) light, in conjunction with a history of trauma to the retina.

\section{Conclusion}

Web queries' mapping provided collateral evidence on the potential causality between HEV light and retinal damage, including macular degeneration, retinal holes and perforations, retinal tears, and potentially an incurring retinal detachment. These retinal pathologies potentiate visual impairment and vision loss across all age groups, including pediatric age groups. Future research on HEV light mandates rigor observational and experimental studies using preclinical and clinical models.

\section{Availability of Data}

Our data, including the raw dataset, are available upon request from the corresponding author.

\section{Conflict of Interest}

The authors declare that there are no conflicts of interest and that they have self-funded the study. 


\section{References}

1. Ouyang X, Yang J, Hong Z, Wu Y, Xie Y, Wang G. Mechanisms of blue light-induced eye hazard and protective measures: A review. Biomedicine \& Pharmacotherapy. 2020; 130: 110577.

2. Tao JX, Zhou WC, Zhu XG. Mitochondria as potential targets and initiators of the blue light hazard to the retina. Oxidative Medicine and Cellular Longevity. 2019: 6435364.

3. The Royal Swedish Academy of Sciences. The Nobel Prize in Physics 2014. https://www.nobelprize.org/prizes/physics/2014/pressrelease/\#: : text $=$ The $\% 20$ Nobel\%20Prize $\% 20$ in $\% 20 P$ hysics $\% 202014 \% 20$ was\%20awarded\%20jointly\%20to \%2Dsaving\%20white\%20light\%20sources. $\% 22$ (accessed 17 April 2021).

4. Leung TW, Li RW, Kee CS. Blue-light filtering spectacle lenses: optical and clinical performances. PloS one. 2017; 12(1): e0169114.

5. Giannos SA, Kraft ER, Lyons LJ, Gupta PK. Spectral evaluation of eyeglass blocking efficiency of ultraviolet/high-energy visible blue light for ocular protection. Optometry and Vision Science. 2019; 96(7): 513-522.

6. Microsoft Corporation. Google Trends. Available from

https://trends.google.com/trends/explore? date $=$ all\&q $=\% 2 \mathrm{Fm} \% 2 \mathrm{FObmsyd} \% 2 \mathrm{Fg} \% 2 \mathrm{Fl} 22 \mathrm{zkl} \quad 4, \% 2 \mathrm{Fg} \% 2 \mathrm{Fl}$ 1c0r5q2m4,\%2Fm\%2F03mmxd,\%2Fm\%2F032l0v (accessed 14 April 2021).

7. Oxford Centre for Evidence-Based Medicine. Oxford Centre for Evidence-Based Medicine: Levels of Evidence (March 2009). Available from https://www.cebm.ox.ac.uk/resources/levels-ofevidenceloxford-centre-for-evidence-based-medicinelevels-of-evidence-march-2009 (accessed 13 April 2021).
8. Al-Imam A, Khalid U, Al-Doori HJ. Predictive Epidemiology for SARS-CoV-2 Pandemic in Iraq. Asian Journal of Medical Sciences. 2021; 12(3): 121-124

9. Al-Imam A, Khalid U, Al-Doori HJ. Clustering Analysis of Coronavirus Disease 2019 Pandemic. Asian Journal of Medical Sciences. 2021; 12(2): 108-113 10. Al-Imam A, Motyka MA, Al-Doori HJ. Surface Web Merits for SARS-CoV-2 Pandemic in Iraq. Journal of the Faculty of Medicine Baghdad. 2020; 62(4): 117127

11. Motyka MA, Al-Imam A, Aljarshawi MHA. SARSCoV-2 pandemic as an anomie. Przestrzeń Spoleczna (Social Space). 2/2020 (20): 111-144.

12.Al-Imam A, Al-Shalchi A. Ekbom's Delusional Parasitosis: A Systematic Review. Egyptian Journal of Dermatology and Venerology. 2019; 39(1): 5-13.

13. Al-Imam A, AbdulMajeed BA. Novel Psychoactive Substances: Systematic Review and Evidence-Based Analysis of Literature. Global Journal of Health Science. 2017; 9(11):1-13.

14.Al-Imam A. A systematic literature review on delusional parasitosis. Journal of Dermatology and Dermatologic Surgery. 2016; 20(1): 5-14.

15. Al-Imam A, AbdulMajeed BA. NBOMe Compounds: Systematic Review and Data Crunching of the Surface Web. Global Journal of Health Science. 2017; 9(11):126-139.

16. Al-Imam A, Abdul-Wahaab IT, Konuri VK, Sahai A, Al-Shalchy AK. Unification of Frequentist Inference and Machine Learning for Pterygomaxillary Morphometrics. Folia Morphologica. 2021; 80(3): 625-641.

17. Al-Imam A, Motyka MA, Jędrzejko MZ. Conflicting Opinions in Connection with Digital Superintelligence. IAES International Journal of Artificial Intelligence. 2020; 9(2): 336-348 


\title{
سلوك البحث عن المعلومات عبر الإنترنت فيما يتعلق بالضوء المرئي عالي الطاقة (الأزرق) في طب العيون
}

\author{
د. أحمد محمدلطفي محمود الامام

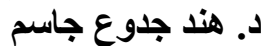

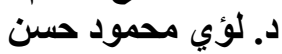

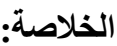

خلفية البحث: الضوء المرئي عالي الطاقة (HEV) يمثلاك ترددًا عاليًا في النطاق البنفجي و الأزرق من طيف الضوء المرئي. للضوء الأزرق صلة بطب

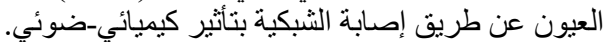

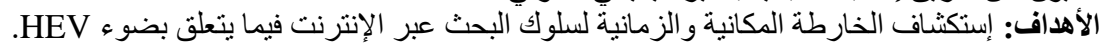

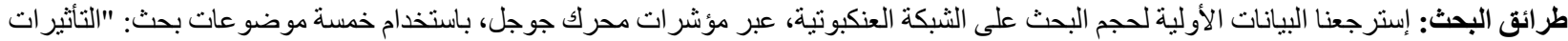

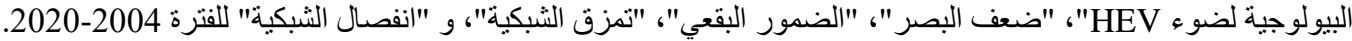

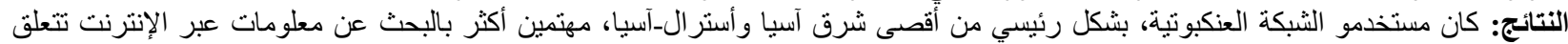

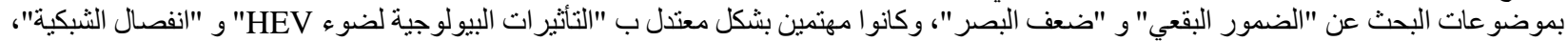

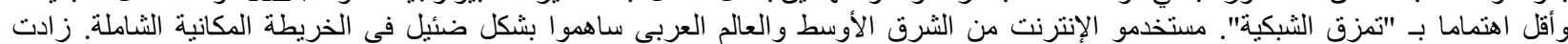

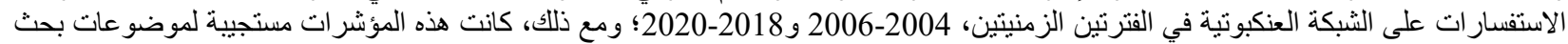

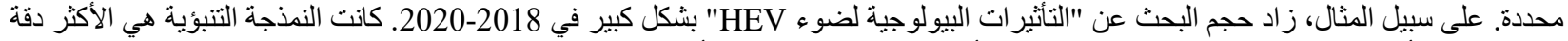

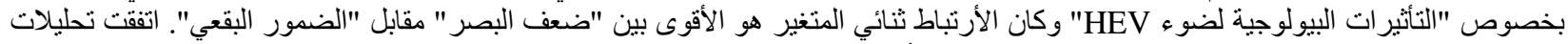

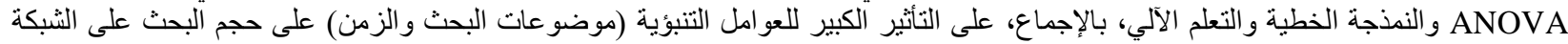
العنكبوتية.

الاستنتاج: قدم تخطيط إستعلامات الثبكة العنكبوتية دليلًا غير مبانشر على العلاقة السبيية بين ضو HEV وتلف الثبكية. يتطلب البحث المستقبلي دراسات

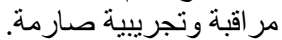
الكلمات المفتاحية: الضوء الأزرق؛ الضوء 